The International Journal of Indian Psychology

ISSN 2348-5396 (e) | ISSN: 2349-3429 (p)

Volume 4, Issue 1, No. 81, DIP: 18.01.126/20160401

ISBN: 978-1-365-59365-9

http://www.ijip.in | October-December, 2016

\title{
Effect of Spousal Appraisal of Identity Consistency on Well-being of Married Females
}

\author{
Mrs. Suchandra Ghosh ${ }^{1 *}$, Prof. Pritha Mukhopadhyay ${ }^{2}$
}

\section{ABSTRACT}

Our research frame work concerned on delineating the relationship between the perceived spousal appraisal of female's Identity Consistency(IC) and its impact on their sense of wellbeing. Identity refers to a person's sense of self as unique being developed out of the various intimate figures and associations of adolescence (Erikson, 1959).

Previous research suggests that consistent self-image across intimate relationships is more valued in western society than eastern society (Heine, 2001; Heine \& Lehman, 1997, 1999; Markus \& Kitayama, 1994; Suh, 2002, English and Chen, 2011). Moreover, contradictory findings have been observed in eastern culture where Shuh (2002) in her study found consistency of Identity is less valued in eastern culture where as Dhar (2010) in her study concluded with reversed findings that Consistency is valued in eastern culture. Shuh (2002) used 20 maximum variability traits to measure one's Identity Consistency (IC) whereas Dhar (2010) used 80 moderate variability traits to measure one's Identity consistency. Hence, It was needed to investigate further this contradictory findings in eastern culture.

The sample comprised of 130 love married couples (non working female partner). They were administered Identity Consistency scale ( Prepared by Dhar,2010) and Subjective wellbeing Scale (Sell and Nagpal, 1992).

Trait Selection for Identity Consistency Scale: For the present study, initially 52 maximum variability traits (like sensitive, talkative etc) along with 80 moderate variability traits from Dhar's study (like anxious, submissive etc) were considered and finally 50 traits were selected with mixed of maximum variability and moderate variability traits to assess Identity Consistency of females for the present study.

\footnotetext{
${ }^{1}$ Research scholar, Department of Psychology, Calcutta University, Kolkata, West Bengal, India

${ }^{2}$ Professor, Department of Psychology, Calcutta University, Kolkata, West Bengal, India

*Responding Author

(c) 2016 Ghosh S, Mukhopadhyay P; licensee IJIP. This is an Open Access Research distributed under the terms of the Creative Commons Attribution License (http://creativecommons.org/licenses/by/2.0), which permits unrestricted use, distribution, and reproduction in any Medium, provided the original work is properly cited.
} 


\section{Effect of Spousal Appraisal of Identity Consistency on Well-being of Married Females}

Internal consistency of each female has been obtained in terms of percentage of variance by using factor analysis by principal component method. Mean, standard deviations ,'t' test Product moment coefficient of correlation and Stepwise Multiple Regression Analysis have been carried out for further analysis with the considered variables in the present study.

Results showed that appraised spousal overestimation of females' IC by females are leading to a satisfactory sense of wellbeing of them may be indicative of the fact that they find the meaningfulness while appraised positively about their inner integrity by 'the person with whom they share the most intimate relation'. However, females' self appraisal of IC is independent on their sense of wellbeing leading to the fact that they are less disturbed by this incongruity.

Keywords: Appraisals of Females’ Identity Consistency, Sense Of Subjective Wellbeing

Humans have to play several roles in their lifetimes but they internalize those which help to form their self-concept (Burke and Tally, 1977). Specially, in eastern collectivist society, 'self' is mainly apprehended to be formed after societal interaction and connectedness with important social intimate relations (Ho, 1993). Erikson described Identity as "a subjective sense as well as an observable quality of personal sameness and continuity, paired with some belief in the sameness and continuity of some shared world image” (Erikson, 1970). With the continuous reciprocal influence in our lives and after exploring latest knowledge about self and others, 'sense of self as unique being' is also altered. But Individuals have preparedness to preserve internal consistency (Erikson, 1959).

People aspire to minimize their unstable mental experiences to retain their sense of wellbeing (Jourand, 1985; Lecky, 1995; Maslow, 1954; Rogers, 1951). Verma and Verma (1989) defined wellbeing as 'a subjective feeling of contentment, happiness and satisfaction with life's experiences and one's role in the world of work, sense of achievement utility belongingness and no distress, dissatisfaction or worry".

According to western psychological theories (e.g., Erikson, 1950; Festinger, 1957; Jourard, 1963; Lecky, 1945; Maslow,1954; Rogers, 1961), mental health and wellbeing can be predicted with 'the degree of the consistency. Though researchers however, in eastern cultures (Heine, 2001; Heine \& Lehman,1997, 1999; Markus \& Kitayama, 1994; Suh, 2002) found, inconsistent relationship between identity consistency and mental health which suggest inconsistencies do not always lead to maladjustments and poor sense of wellbeing of individuals. Perhaps global consistencies are not prioritized in eastern cultures, rather the adjustment with social contexts and nurturing 'relation specific self' are more valued. (English and Chen, 2011) 


\section{Research related Identity Consistency and Subjective well being:}

With the rising value of consistency regarding identity, researchers apply different methods to assess consistency. In one study Fitch and Adams (1982) applied Ego-identity interview to assess the identity-intimacy relationship on 28 males and 50 females, in another study Objective measure of ego identity status were assessed using likert scale comprised of 24 items (Adams, Shea and Fitch,1979) .

Whitbourne (1986) conducted a semi structured Adult Identity Interview where the participants were asked to narrate their identities regarding their current employment and family conditions for assessing Identity flexibility ,that is the mindset of individuals to look over different identity commitments in the field of family and work, on a sample of 57 adults. Identity flexibility was significantly related to life change and openness to experience, signifying that both personality and appraise and real social assets are predictors of 'flexibility and life change' in adulthood. Suh (2002) attempted to study the relevance of Identity Consistency (IC) to the culture since consistency among different aspects of the identity is emphasized in the western culture \& multiple selves are often viewed as co-existing realities in the East Asian culture (Korean). To obtain an index of identity consistency (IC) level, participants were asked to rate 25 personality traits that described his or her "general self" on a scale ranging from 1 (not at all like myself) to 7 (very much like myself). Afterwards, each personality trait was rated across 4 relationships (father, mother, romantic partner and strangers). The 25 adjectives consisted of positive (e.g., cheerful), neutral (e.g., serious), and negative (e.g., cynical) personality characteristics were compiled in reference to the Big Five traits (Goldberg, 1993). After converting each person's 125 ratings into a $25 \mathrm{X} 5$ matrix (25 traits in five different contexts, including the general self), On the basis of this statistical logic, the percentage of variance accounted for by the first factor was used as a personal index of IC. Results indicated that compared with Western culture, Korean's are more flexible across social relations and their subjective wellbeing was less predictable from levels of Identity Consistency.

However, Dhar et.al (2010) studied the relationship between Identity Consistency (IC) and General Well-Being (GWB) of the college students of Kolkata. To assess IC index of the participants, they referred Suh's scale on IC (2002).

The 20 traits included in their study were as follows: Emotional, Modest, Friendly, Co-operative, Talkative, Impatient, Impulsive, Open-minded, Out-going, Introverted, Dominant, Business-like, Calculative, Honest, Twofaced, Cheerful, Kind, Rational, and Cranky. They found that Identity consistency is positively associated with wellbeing of the college and university students of Kolkata, aged between 18 to 25 years. Internal consistency of each individual was obtained in terms of percentage of variance by using factor analysis by principal component method. The percentage of variance scores were then transformed into IC scores through angular transformation. The sample was administered IC scale in order to obtain an index of IC and 


\section{Effect of Spousal Appraisal of Identity Consistency on Well-being of Married Females}

GWB was assessed using the PGI GWB Scale (Verma \& Verma 1989). Results indicated that IC has a significant positive correlation with GWB and it contributes GWB signifying consistent identity across intimate relations can be said to be a prerequisite of mental health leading to satisfactory perception of well being.

English \& Chen (2011) attempted to study the impact of self-concept consistency across European American and East Asian American (i.e., Chinese, Japanese, and Korean American) cultures to assess the consistency across relationship contexts and subjective authenticity and the consistency within relationship over time. 18 different personality traits (anxious, bossy, careless, considerate, cooperative, expressive, friendly, insecure, kind, lazy, moody, openminded, organized, polite, responsible, self confident, shy, talkative) using a 7-point scale (1 = not at all,7 = very much) were administered to the participants over three intimate relationships, namely, self with friend, self with mother, and self with romantic partner.

To assess Index of inconsistency across relationships, the standard deviation of each participant's self descriptiveness ratings for each trait across his or her relationships was computed and then averaged the standard deviations across the traits. Larger standard deviations indicate greater inconsistency in participants' self-views across different relationship. Result suggests that inconsistency of trait self-perceptions across different relationship contexts was linked to lower subjective authenticity and relationship quality for European Americans but not East Asian Americans. However, inconsistency within the same relationship context over time showed smaller level of authenticity and relationship quality among both the cultures.

Banerjee (2005) studied self view of men and women, the consistency of self view across different relations and relations between IC, marital intimacy and quality of marriage. Here, , the respondents were requested to give 10 accurate personality traits of themselves and rated it on a 5 point scale ranging from 1 to 5 across 4 intimate relations (father, mother, spouse and same gender friend) to assess IC. IC index was obtained computing factor analysis and $1^{\text {st }}$ factor was considered as IC score.

Dhar (2010, 2015) studied on Identity Consistency, quality of marital life and attachments. Sufficient numbers of traits were collected from 520 individuals and the traits were rated by 100 judges on the basis of its variability across different intimate relations. 80 traits which had moderate variations across relations were selected for the study. IC of the individuals were determined administering 80 moderate variability personality traits on subjects and they were asked to rate it on a 5 point scale on the basis of its expression on 4 intimate relations (father, mother, same gender friend). IC index was determined after angular transformation of $1^{\text {st }}$ factor of factor analysis. 


\section{Effect of Spousal Appraisal of Identity Consistency on Well-being of Married Females}

The studies mentioned so far focus that fact that though several studies have been conducted to find different angles of relationships among formation of a consistent identity and its relations to concerned variables in intimate relations but research has yet to explore impact of spousal appraisal regarding other partner's consistency across relations on their sense of wellbeing. Hence, the study has taken into consideration to conceptualize the fact that how females apprehend about their true and presented self and how the difference between the two selves affect the sense of wellbeing of females. We are considering the perception of the most intimate relation that is Spousal relation in adult life which may have a strong impact on females' cognitive process.

Henceforth, the current study aims to explore Identity Consistency Index for females of both appraisals.

For the present study, two appraisals have been taken into consideration.

$1^{\text {st }}$ appraisal- self appraisal of females regarding their identity consistency

$2^{\text {nd }}$ appraisal- Females' perception about their spouse' appraisal regarding females' identity consistency

The study also attempts to explore whether the identity consistency of females have any contribution to the subjective wellbeing of the females. At the same time the study also discern whether the differences of identity consistency as viewed by self and their spouse have any contribution to sense of subjective wellbeing of married women.

\section{METHODOLOGY}

\section{Objective}

A) Selection of the 50 traits or personality characteristics of individual those are varied across different intimate relations (among 132 moderate and maximum variability traits of Dhar's Study).

B) To explore

1) whether there is any significant difference between $1^{\text {st }}$ appraisal of Identity Consistency (self appraisal of Identity Consistency) and 2nd appraisal of Identity Consistency (perceived spousal appraisal of Identity Consistency).

2) Identity Consistency index of 130 married females along with their Sense of subjective wellbeing.

3) whether there is difference in sense of subjective wellbeing between females who underestimated by their spouses regarding their IC than the females who overestimated by their spouse.

4) Whether these differences in perceived Identity consistency have contribution to the females' sense of wellbeing

5) The relative contribution of Identity Consistency of both appraisals (self and spousal appraisal) to the females' sense of wellbeing. 


\section{Sample}

The sample comprised of a group of individuals which included 130 married females and 130 married males (130 married couples) selected through purposive sampling from the society (Indian culture specifically Bengali Culture).

\section{Inclusion Criteria}

1) Minimum educational qualification is graduate.

2) Age range within 25-35 years.

3) Living in nuclear or extended nuclear family.

4) Individuals who do not have history of divorce or separation (not remarried).

5) Minimum five years duration of marriage.

6) Both the parents and in-laws are alive.

7) The spouse is alive.

8) Individuals staying with their spouse at present.

9) Convenient in English language.

\section{Tools Used}

1) Socio demographic data sheet.

2) Kuppuswamy's Socioeconomic Status Scale(modified version by Gururaj and Maheshwaran 2014)

3) General Health Questionnaire ( Goldberg, 1979)

4) Identity Consistency scale (Dhar,2010)

5) Subjective wellbeing scale (Sell ad Nagpal, 1992)

\section{Socio Demographic Data sheet}

It consists of personal information about the subject regarding age, sex, education, occupation, income, family type, type of marriage, duration of marriage, number of children and whether the subject had any past or current history of psychiatric illness or only history of availing counseling or psychotherapeutic services.

\section{Kuppuswamy's Socioeconomic Status Scale}

It assess the socio-economic status of an individual.

3. General Health Questionnaire-Form 28 (Goldberg, 1979) as Screening tool It is a selfadministrative questionnaire consisting 28 items which was used as screening tool to detect the psychiatric distress in the subjects. Those who have crossed the cut off score (4 out of 28), was not considered for the present study.

\section{Identity Consistency Scale (Dhar, 2010)}

To obtain an index of Identity consistency, E.M Suh's study on culture, identity consistency and subjective wellbeing (Suh, 2002) and Dhar's study of IC, Attachment and Marital quality in Indian adults (2010) were taken as reference. 


\section{Effect of Spousal Appraisal of Identity Consistency on Well-being of Married Females}

a) 50 traits were selected from 132 moderate and maximum variability traits (Dhar,2010). The respondents were asked to choose 10 traits of themselves from this 50 traits list which best describe themselves across six intimate relations (relationship with father, relationship with mother, relationship with spouse, relationship with same gender friend, relationship with motherin-law, relationship with father-in law)

b) They were asked to rate the expression of trait or characteristics across these six relations on a 5 point scale, where

$1=$ Maximum expression of the traits / characteristics

2 = Much expression of the traits / characteristics

$3=$ Moderate expression of the traits / characteristics

$4=$ Less expression of the traits / characteristics

5 = Minimum expression of the traits / characteristics

\section{Instruction for Identity Consistency(IC) Scale}

1. Regarding the first appraisal, the instruction of the Identity Consistency Scale as such is applicable

Out of the given fifty traits, you have to choose ten traits that describes you best and which you perceive as being related to these six intimate relationships i.e., (father, mother, spouse, same gender friend, father-in-law, mother-in-law ).Please rate the relevance of the following traits or characteristics as they are expressed in people in 6 relations ,i.e., father, mother, spouse, same gender friend, father-in-law, mother-in-law on a five point scale where

1. Maximum expression of the trait or characteristics

2. Good expression of the trait or characteristics

3. Moderate expression of the trait or characteristics

4. Less expression of the trait or characteristics

5. Minimum expression of the trait or characteristics

2. Regarding $2^{\text {nd }}$ appraisal : In case of female's perception of her spouse's appraisal of her own Identity Consistency (2nd appraisal) the following instruction has been given :

There are self-chosen 10 individual's psychological characteristics or traits which described you best in people in 6 relations, i.e., father, mother, spouse, same gender friend, father-in-law, mother-in-law. "If your husband is asked how you view your wife in following traits across these six relationship context (i.e., father, mother, spouse, same gender friend, father-in-law, motherin-law), what will be his ratings regarding you from the given five point scale?” There is no right or wrong answer and be sure that you have responded to all the traits. where

1. Maximum expression of the trait or characteristics

2. Good expression of the trait or characteristics

3. Moderate expression of the trait or characteristics

4. Less expression of the trait or characteristics

5. Minimum expression of the trait or characteristics 


\section{Scoring and interpretation of the data have been done in two categories.}

$1^{\text {st }}$ Appraisal- Females' appraisal of her own Identity Consistency

$2^{\text {nd }}$ Appraisal-Female's appraisal about her spouse's appraisal about her Identity Consistency.

Difference Score: Difference between $1^{\text {st }}$ and $2^{\text {nd }}$ appraisal of Identity Consistency

Overestimation: If $2^{\text {nd }}$ appraisal is greater than $1^{\text {st }}$ appraisal of females' Identity Consistency Other Types of difference score related overestimation

ICO1 denotes the group of females who differ maximally from their spouses regarding their IC

ICO2 denotes the group of females who differs minimally from their spouses regarding their IC

Underestimation: If 1st appraisal is greater than 2nd appraisal of females' Identity Consistency Other Types of difference score related underestimation

ICU1 denotes the group of females who differs maximally from their spouses regarding their IC ICU2 denotes the group of females who differs minimally from their spouses regarding their IC

\section{Subjective wellbeingness (Sell and Nagpal, 1992)}

It is designed to measure feeling of wellbeing or ill-being as experienced by individual or a group of individuals in various day to day life concerns. It consists of 40 items with 11 dimensions.

\section{Statistical analysis}

The results have been calculated on the basis of SPSS-12

1) A data matrix was constructed for each 260 people (130 couples) on the basis of the 10 traits rated by them across 6 intimate relations.

2) Internal consistency of each person has been obtained in terms of percentage of variance using factor analysis by principal component method.

3) The percentage of variance scores has been transformed into IC indices through angular transformation.

4) Descriptive Statistics (mean and standard deviations) has been computed for Identity Consistency Scale for $1^{\text {st }}$ and $2^{\text {nd }}$ appraisal, Sense of Subjective wellbeing.

5) ' $t$ ' test has been used to determine whether the self-appraisal and perceived spousal appraisal of Identity Consistency of females differ significantly or not.

6) Product moment coefficient of correlation has been used to find out the relationships between Identity Consistency and subjective Wellbeing.

7) Stepwise Multiple Regression Analysis (SMRA) was done to find out relative contribution of Identity Consistency in the development of quality of Sense of subjective wellbeing. 


\section{RESULT}

t-test

Table1a: Mean and Standard deviation of both appraisals $\left(1^{\text {st }}\right.$ and $\left.2^{\text {nd }}\right)$ of Identity Consistency (IC) and sense of subjective wellbeing (SWB) of females as well as t-test between $1^{\text {st }}$ and $2^{\text {nd }}$ appraisal of IC

\begin{tabular}{|l|l|l|l|l|}
\hline & $\mathbf{N}$ & Mean & SD & Sig \\
\hline ICF & 130 & 61.52 & 14.81 & .01 \\
\hline ICFM & 130 & 65.02 & 15.25 & \\
\hline SWB & 130 & 91.39 & 9.86 & \\
\hline
\end{tabular}

$\mathrm{SWB}=$ Sense of Subjective Wellbeing

$\mathrm{ICF}=1^{\text {st }}$ appraisal that is self appraisal of females' Identity consistency across various social roles (i.e, father, mother, spouse, same-gender friend, father in law and mother in law). Example: if we are considering 'sensitive' as self-chosen trait by one of the participants, I ask her to rate how much 'sensitive' she is towards the following relationship contexts (with her father, with her mother, with her spouse, with her friend, with her father-in-law and with her mother-in-law)

ICMF $=2^{\text {nd }}$ appraisal of Identity consistency of females. Spouses' appraisal of females' as perceived Identity consistency by females across various social roles (i.e, father, mother, spouse, same-gender friend, father in law and mother in law).

Example: "If I ask your husband to rate you on how much 'sensitive' you are towards the following relationship contexts (with her father, with her mother, with her spouse, with her friend, with her father-in-law and with her mother-in-law), what will be his response?”

The above findings depicted the nature of the sample regarding Identity Consistency and sense of subjective wellbeing considered for the present study. As far as the Identity Consistency of the total group is concerned, it is evident from the obtained result that sample has average consistencies regarding their identities and perceived identities. These two appraisals regarding their identities differ significantly from each other; where self or $1^{\text {st }}$ appraisal (ICF) is significantly smaller in Identity Consistency than $2^{\text {nd }}$ appraisal (ICFM). It is also evident from the above findings that sample has satisfactory sense of subjective wellbeing.

Table 1b: (comparison between underestimation and overestimation)

\begin{tabular}{|l|l|l|l|l|l|l|l|}
\hline & N & sig & $\begin{array}{l}\text { Mean of } \\
\text { SWB }\end{array}$ & SD & $\begin{array}{l}\text { Sig } \\
\text { (SWB) }\end{array}$ & t & df \\
\hline Overestimation & 72 & .01 & 90.87 & 12.04 & .037 & 2.11 & 125 \\
\hline Underestimation & 55 & & 86.25 & 12.43 & & & \\
\hline
\end{tabular}

Overestimation $=\mathrm{ICMF}>\mathrm{ICF}$

Underestimation $=\mathrm{ICMF}<\mathrm{ICF}$

SWB=sense of subjective wellbeing

(C) The International Journal of Indian Psychology, ISSN 2348-5396 (e)| ISSN: 2349-3429 (p) | 54 


\section{Effect of Spousal Appraisal of Identity Consistency on Well-being of Married Females}

The above findings from the table signifies that females who perceived to be underestimated by their spouse regarding their Identity consistency differ significantly In their IC as well as SWB from females who perceived to be overestimated by their spouse regarding their Identity Consistency. Those who overestimated by their spouses regarding their identity consistency showed significantly better sense of subjective wellbeing than those who were underestimated by their spouse.

Table 1c: (Comparison between ICU1 and ICU2)

\begin{tabular}{|l|l|l|l|l|l|l|}
\hline & $\mathbf{N}$ & $\begin{array}{l}\text { Mean of } \\
\text { SWB }\end{array}$ & SD & Sig & $\begin{array}{l}\text { Man } \\
\text { whitney }\end{array}$ & Wilcoxon \\
\hline ICU1 & 13 & 84.92 & 12.16 & .650 & 75 & 166 \\
\hline ICU2 & 13 & 86.92 & 12.73 & & & \\
\hline
\end{tabular}

ICU1= The females who maximally differ from their spouses' view regarding females' Identity consistency

ICU2= The females who minimally differ from their spouses' view regarding females' Identity consistency

SWB=sense of subjective wellbeing

The above findings from the table deals with the groups of females who perceived to be underestimated by their spouse regarding their identity consistency. The result signifies that females who perceived to be maximally differ from their spouses' view regarding females' Identity consistency (ICU1), does not differ significantly in sense of subjective wellbeing from females who perceived to be minimally differ from their spouses' view regarding their Identity consistency (ICU2). Though mean of SWB shows better sense of subjective wellbeing in ICU2 than ICU1 signifying that females who perceived to be minimally differ from their spouses' view regarding females' Identity consistency, have better sense of wellbeing than the females who perceived to be maximally differ from their spouses' view regarding females' Identity consistency.

Table 1d: (Comparison between ICO1 and ICO2)

\begin{tabular}{|l|l|l|l|l|l|}
\hline & $\mathbf{N}$ & $\begin{array}{l}\text { Mean of } \\
\text { SWB }\end{array}$ & SD & Sig & Man whitney \\
\hline ICO1 & 16 & 89.31 & 13.08 & .574 & 127 \\
\hline ICO2 & 18 & 92.5 & 9.81 & & \\
\hline
\end{tabular}

ICO1=The females who maximally differ from their spouses' view regarding females' Identity consistency

ICO2=The females who minimally differ from their spouses' view regarding females' Identity consistency

SWB=sense of subjective wellbeing 


\section{Effect of Spousal Appraisal of Identity Consistency on Well-being of Married Females}

The above findings from the table deals with the groups of females who perceived to be overestimated by their spouse regarding their identity consistency. The result signifies that females who perceived to be maximally differ from their spouses' view regarding females' Identity consistency (ICO1), does not differ significantly in sense of subjective wellbeing from females who perceived to be minimally differ from their spouses' view regarding their Identity consistency (ICO2). Though mean of SWB shows better sense of subjective wellbeing in ICO2 than ICO1 signifying that females who perceived to be minimally differ from their spouses' view regarding females' Identity consistency, have better sense of wellbeing than the females who perceived to be maximally differ from their spouses' view regarding females' Identity consistency.

Table 2 : Correlations of Subjective wellbeing, Identity consistency of the females $\left(1^{\text {st }}\right.$ appraisal and 2nd appraisal of Identity consistency)

\begin{tabular}{|l|l|l|l|l|}
\hline \multirow{3}{*}{ SWB } & & SWB & ICF & ICMF \\
\cline { 2 - 5 } & Pearson Correlation & 1 & .069 & $.240^{* *}$ \\
\cline { 2 - 5 } & Sig. (2-tailed) & & .434 & .006 \\
\cline { 2 - 5 } & $\mathrm{N}$ & 130 & 130 & 130 \\
\hline \multirow{3}{*}{ ICF } & Pearson Correlation & .069 & 1 & $.420^{* *}$ \\
\cline { 2 - 5 } & Sig. (2-tailed) & .434 & & .001 \\
\cline { 2 - 5 } & $\mathrm{N}$ & 130 & 130 & 130 \\
\hline \multirow{3}{*}{ ICMF } & Pearson Correlation & $.240^{* *}$ & $.420^{* *}$ & 1 \\
\cline { 2 - 5 } & Sig. (2-tailed) & .006 & .000 & \\
\cline { 2 - 5 } & $\mathrm{N}$ & 130 & 130 & 130 \\
\hline & & 130 & & \\
\hline
\end{tabular}

** means significant beyond 0.01 level

$\mathrm{SWB}=$ Sense of Subjective Wellbeing

$\mathrm{ICF}=1^{\text {st }}$ appraisal or self appraisal of females Identity consistency across various social roles (i.e, father, mother, spouse, same-gender friend, father in law and mother in law)

ICMF $=2^{\text {nd }}$ appraisal of Identity consistency of females or Appraised spouses' appraisal of females’ Identity consistency by females.

The above table reveals that there is significant correlations between sense of subjective wellbeing and $2^{\text {nd }}$ appraisal of IC(ICMF). This indicates that Sense of subjective wellbeing is related to $2^{\text {nd }}$ appraisal of identity consistency of females. This findings indicate that with an increase in females' consistency regarding expressions of traits or characteristics across intimate relationships of father, mother, same gender friends, father in law and mother in law), there is also an increase in positive perception regarding sense of subjective wellbeing. 


\section{Effect of Spousal Appraisal of Identity Consistency on Well-being of Married Females}

However, 1st appraisal or self appraisal of identity consistency did not show any significant relation to sense of subjective wellbeing of females may be indicative of the fact that females' sense of subjective wellbeing is independent of females' self appraisal of her IC.

\section{Regression}

\begin{tabular}{|l|l|l|l|l|l|}
\hline $\begin{array}{l}\text { Dependent } \\
\text { Variable }\end{array}$ & $\begin{array}{l}\text { Predictor } \\
\text { variables }\end{array}$ & $\begin{array}{l}\text { Adjusted } \\
\text { Square }\end{array}$ & F & $\begin{array}{l}\text { Significance } \\
\text { level }\end{array}$ & $\begin{array}{l}\text { Beta } \\
\text { coefficient }\end{array}$ \\
\hline SWB & ICMF & .05 & 7.81 & .006 & .240 \\
\hline
\end{tabular}

$\mathrm{SWB}=$ Sense of Subjective Wellbeing

ICMF $=2^{\text {nd }}$ appraisal of Identity consistency of females or Appraised spouses' appraisal of females' Identity consistency by females.

It was also observed from table 1.3 that ICMF alone predicted the variance of SWB by 5\%. The positive $b$ values suggest the positive impact of ICMF on the aspects of Sense of subjective wellbeing.

\section{DISCUSSION}

The present study on Identity Consistency and subjective wellbeing in married females are guided by the Eriksonian analysis on ego identity which he conceptualized as continuity of images of selfhood that revise throughout the life cycles (Erikson, 1968). It may be noted here that despite the various changes in different aspects of one's life and relations, somehow one maintains or strives to be the same person and wished to be viewed as same individual by significant others. The present study attempts to assess the impact of significant others' (spouse) view of females' identity on their sense of wellbeing.

The objective of this study is therefore to explore how females' view themselves with respect to their close relationships and how they view about their spouses' appraisals regarding them in these close relationships; how these two views related to the sense of subjective wellbeing of females.

The present study was a cross sectional in nature and was done in two stages where first stage deals with the selection of the traits or characteristics of individuals from 132 traits of Dhar's study (2010) and assessment of Identity Consistency of married females.

Second stage deals with the associations of the different appraisals ( $1^{\text {st }}$ and $2^{\text {nd }}$ appraisals) of identity consistency of married females and their sense of subjective wellbeing. 


\section{Effect of Spousal Appraisal of Identity Consistency on Well-being of Married Females}

\section{First Stage:}

\section{A) Selection of traits and Assessment of IC}

\section{Steps 1: Selection of traits to assess IC index of the females's}

Dhar (2010) has given more priority to moderate variability traits and avoid maximum variability traits as it may lead to the person inconsistency in nature. In her study on Identity consistency, Attachment and Marital quality in Indian adults, she used 80 moderate variability from collected 200 self described traits. Where as from Suh's study (2002) it can be observed that she has given more priority to maximum variability traits as these traits vary maximally across different relations. In that study she choose 20 most frequently occurring personality traits which describe the subjects best, collected from two different culture (U.S and Korea, ten traits for each culture) and maximum variability traits like talkative were given more priority over minimum / moderate variability traits which are more consistent personality traits of an individual or innate nature of an optimistic and does not vary depends on different socio relations.

Taking in to consideration this contrary views of researchers from eastern and western cultures, it was initially decided to include totals 132 traits (52 maximum variability and 80 minimum variability) for the present study and let the subjects choose freely according to their characteristics being it moderate or maximum, but it was found that subjects were confused and facing difficulties to choose 10 best self-described traits among 132 traits. Moreover, it was noted that though the traits chosen by Dhar (2010) have moderate variability, the range of average number of variation lies between 1.05 to 2.29. Among 80 moderate variability traits used in Dhar's study, the average number of variation for 61 traits are below 2. Average variation 1 means it does not vary across social intimate relations, i.e., father, mother, spouse and same gender friend. It means that 61 traits selected by Dhar's study did not vary across the intimate relations as rated by 100 judges in her study. So, it was decided to include maximum variability traits which have the average variation ranging from 2.30 to 3.13 in Dhar's study where average variation 2 means the traits vary in at least one relations among 4 intimate relations, i.e., father, mother, spouse and same gender friend and average variation 3 means the traits vary across 2 relations among 4 intimate relations.

After a pilot study with 35 females, it was found that these large number of traits(132 traits) are confusing to the subjects; some traits are some what similar or related meanings like silent-calm, cooperative-adjustable, Dutiful-responsible, Touchy-Impulsive, Loving-Affectionate etc. To reduce this difficulty and avoid these undesired circumstances, it was decided to include 50 traits will be chosen for the present study and among 50 traits subjects have to select ten traits those describe them best.

The final randomized 50 traits for the present study are: Sensitive, Illogical, Moody, Harsh, Critical, Obedient, Honest, Talkative, Loyal, Arrogant, Attentive, Cooperative, Responsible, Strict, Forgiving, Supportive, Submissive, Protective, Analytic, Sincere, Dominant, Impatient, 
Effect of Spousal Appraisal of Identity Consistency on Well-being of Married Females

Open-minded, Frank, Nagging, Dependant, Caring, Disturbing, Helpful, Active, Faithful, Aggressive, Anxious, Defensive, Emotional, Manipulative, Rigid, Truthful, Serious, Devoted, Predictable, Outspoken, Assisting, Good-listener, Non-expressive, Sacrificing, generous, Dutiful, Possessive, Modest.

\section{Step 2: Assessment of IC}

The identity consistency indices of 130 married female were calculated on the basis of the following steps using SPSS 12.0.

a) A data matrix was constructed for each of 130 married females on the basis of 10 traits rated by them across 6 intimate relationships. Each females' rating themselves of the 10 traits or characteristics with respect to 6 relationships (Father, Mother, spouse, same gender friend, Father-in-law, Mother-in-law) were arranged in a 10 X 6 matrix (ten self descriptions X sis relationships).

b) Internal consistency of each female was obtained in terms of percentage of variance by using factor analysis. Each individual's matrix was factor analyzed, using the principal component method. The statistical logic used was that, if a person views himself or herself consistently across all intimate relationships, the principal component factor obtained from the within subject factor analysis would account for a large percentage of the persons view variance across relationships and vice versa.

c) The percentage for variance score accounted for by the first factor was transformed into interval scale score using angular transformation and this score was considered as the measure of Identity Consistency ( across relationship ) i.e., the Identity Consistency Index for each female. Percentage of variance will be changed into proportion i.e., if \% of variance is 54.49, proportion will be 0.5449 or 0.54 .

\section{B) Stage 2: Relations between $1^{\text {st }}$ and $2^{\text {nd }}$ appraisal of IC of females and their sense of subjective wellbeing}

"Identity is the accrued confidence that one's ability to maintain inner sameness and continuity...”(Erikson,1959). In the present study it has been operationalized as -person's sense of same ness in various self-described traits across intimate relations-father, mother, spouse, same gender friend, father-in-law and mother-in-law).

It is evident from table1.1 that $2^{\text {nd }}$ appraisal (perceived spouses' appraisal of females) of Identity consistency has significant difference than the $1^{\text {st }}$ appraisal (self appraisal of females) of Identity consistency. An overestimation of IC has been observed in $2^{\text {nd }}$ appraisal of IC in comparison to the $1^{\text {st }}$ appraisal of IC signifying females' inner desire to be appraised as consistent by their spouse (1b). This finding is substantiated from the significant positive correlation between $2^{\text {nd }}$ appraisal of IC and sense of subjective wellbeing of females (Table 2). When females appraise that their husbands perceive them as more consistent in self-described traits across intimate relations as compared to the females' version about themselves, it may appear to the females as a 
Effect of Spousal Appraisal of Identity Consistency on Well-being of Married Females

respectful evaluation by the spouses for them, leading to significantly better sense of subjective wellbeing of the females. It is not perceived as falsified statement but as unconditional acceptance of the spouse in spite of their wives' humane limitation.

The findings substantiate the females' expectation to be positively appraised by the spouse on Identity Consistency as the positive ' $b$ ' value in regression analysis (Table 3) also suggestive of positive contribution of $2^{\text {nd }}$ appraisal of IC on the aspects of Sense of subjective wellbeing. It probably is because of the fact that when females are appraised positively about their inner integrity by 'the person with whom they share the most intimate relation', they find it rewarding and worth that positively affect sense of wellbeing of females. This assures them a feeling of security that gives them a sense of comfort and finally enhancing sense of wellbeing. Since this support is received from the most inmate relationship in their lives, it enhances the maximum feeling of connectedness with their husbands. This connectedness increases SWB not only because their IC has been overestimated by their spouse but this 'connectedness with significant others' help to form females' vision about their self hood (Cross \& Madson, 1997; Diedrick, 1988) by actually increasing their own IC. Supporting this views Markus and Kitayama (1991) also believed that in collectivist society, person is perceived as rooted in a societal framework of interpersonal intimacy and involvement which helps to form and describe 'self.'

From Table 1c and 1d, it is observed that both case of appraisals by females, be it overestimation or underestimation of IC by spouses, there is no significant mean difference of SWB with 'females who appraised to be maximally differ from their spouses' views regarding their IC'(ICMa) and 'females who appraised to be minimally differ from their spouses'(ICMi) views regarding their IC'. Though a trend has been found from 'means' of having better sense of SWB in ICMi than ICMa. So, the 'overestimated or underestimation' is valued over 'maximum and minimum’ discrepancies between opinions.

Though surprisingly from table 2 it has been noted that, being consistent is not prioritized by the females' self view of IC and is not significantly related to the sense of subjective wellbeing of females. It may be because of the fact that females in the present study, being in part of Eastern Asian Culture, have tolerance towards incongruity between personal desire and actual manifestation of behavior. Few research support this fact like- in East Asian culture, unlike western cultures, does not assume to this fact that 'action should be consistent with inner thinking' so they are less affected by cognitive dissonant situations (Heine and Lehsar,1997; Kashima, Seigal, Janaka \& Kashima 1992). Where as Fu et al, (2001) found East Asians are less critical of incongruent acts displayed between private and public situations. So, it can be concluded that females desire to be positively appraised by their spouse regarding their IC though they themselves actually are not so consistent across relations. They are less disturbed by this incongruity so it does not adversely affect their sense of wellbeing supporting the fact that SWB are not related to ICF that is their self appraisal of IC. 


\section{CONCLUSION}

Perceived spousal overestimation of females' IC is well received by the females, leading to a satisfactory sense of wellbeing. However, females' self appraisal of IC and SWB is independent of each other owing to non-maintenance of continuity of self described attributes across the relation fails to maintain continuity which is not disturbed by them.

\section{Acknowledgments}

The author appreciates all those who participated in the study and helped to facilitate the research process.

\section{Conflict of Interests}

The author declared no conflict of interests.

\section{REFERENCE}

Adams, G.R., \& Fitch, S.A. (1982). Ego stage and identity status development: a crosssequential analysis. Journal of Personality and Social Psychology, 43, 574-583.

Adams, G.R., Shea, J., \& Fitch, S.A. (1979). Toward the development of an objective assessment of ego-identity status. Journal of Youth and Adolescence, 8, 223-237.

Banerjee, P. (2005).Identity Consistency, Intimacy and Quality of Marriage in Married Men and Women. Unpublished Dissertation in the partial fulfillment for the M.Phil Degree in Clinical Psychology, Bangalore :NIMHANS.

Burke,Peter J. And Judy Tally, 1977. “The Measurement of Role Identity.” Social Forces 4:881897. City of Chicago.1983.Homelessness in Chicago. Chicago: Social Services Task Force.

Cross, S. E., \& Madson, L. (1997). Models of the self: Self-construals and gender. Psychological Bulletin, 122, 5-37

Dhar,S. and Basu,S.(2009). Identity Consistency and Its relationship with attachment styles and quality of marital life. Indian Institute of Psychometry,23(1), 26-43

Dhar,S.(2010).A study of Identity consistency, attachment styles and qulity of marital life in married women and men. Thesis submitted and awarded for the degree of doctor of philosophy (Science) to the Dept. of Psychology, University of Calcutta: Kolkata

Dhar,S.,Sen,P. and Basu, S.(2010).Identity consistency and general well-being in college students. Psychological Studies.55(2),144-150

Dhar,S.(2015). Identity Consistency, Attachment and Marital Quality in Indian Adults. Psychological Studies. 60(2),239-243

English,T. \& Chen, S.(2011). The self concept consistency and culture: The differential impact of two forms of consistency .Personality and Social Psychology Bulletin XX(X) 1-12.

Erikson, E. H. (1950). Childhood and society. New York, NY: Norton.

Erikson, E.H. (1959). Growth crisis and the healthy personality in identity and the life-cycle. Psychological Issues, 1:50-100. 


\section{Effect of Spousal Appraisal of Identity Consistency on Well-being of Married Females}

Erikson, E.H. (1968). Identity: Youth and Crisis. New York : Norton.

Erikson, E.H. (1970). Reflections on the dissent of contemporary youth., International Journal of Psychoanalysis, 51, 11-22.

Festinger, L. (1957). A theory of cognitive dissonance. Oxford, England: Row, Peterson.

Fu, G.K., Cameron, C.A. and Xu, F. (2001). Chinese and Canadian adults categorization and evaluation of lie and truth telling about prosocial and anti social behavior. Journal of Cross-cultural psychology, 32, 720-727.

Goldberg,D.P. and Hiller, V.F (1979). A scaled version of the general health questionnaire .Psychol Med, 9(1):139-45

Heine, S. J. (2001). Self as a cultural product: An examination of East Asian and North Americans selves. Journal of Personality,69, 881-906.

Heine, S. J., \& Lehman, D. R. (1997). Culture, dissonance, and self-affirmation. Personality and Social Psychology Bulletin,23, 389-400.

Heine, S. J., \& Lehman, D. R. (1999). Culture, self-discrepancies, and self-satisfaction. Personality and Social Psychology Bulletin,25, 915-925.

Heine,S.J, , \& Lehman ,D.R. (1997).Cultural ,dissonance and self affirmation. Journal of personality and social psychology 72 (6), 1268.

Ho, DF. (1993). Relational orientation in Asian social psychology. In U Kim \& JW Berry (Eds.), Indigenous Psychologies: Research and Experience in Cultural Context (pp 240-259), Newbury Park, CA: Sage.

Jourard, S. M. (1963). Personal adjustment: An approach through the study of healthy personality. New York, NY: Macmillan.

Kashima, Y., Kashima, E., Farsides, T., Kim, U., Strack, F., Werth, L., \& Yuki, M. (2004). Culture and context-specific self: The amount and meaning of context-sensitivity of phenomenal self differ across cultures. Self \& Identity, 3, 125-141.

Lecky, P. (1945). Self-consistency: A theory of personality. New York: Island Press.

Markus, H. R., \& Kitayama, S. (1994). A collective fear of the collective: Implications for selves and theories of selves. Personality and Social Psychology Bulletin, 20, 568-579.

Markus, H.R. and Kitayama, S. (1991). Culture and Self: Implications for cognition, emotion and motivation. Psychological Review, 98, 224-253.

Martin, P., Hagestad, G.O., \& Diedrick, P. (1988). Family stories: Events (temporarily) remembered. Journal of Marriage and the Family, 50, 533-541

Maslow. A. H. (1954).Motivation and personality. New York: Harper Brothers.

Rogers, C. (1961). On becoming a person: A therapist's view of psychotherapy. Boston, MA: Houghton Mifflin.

Rogers, Carl (1951). Client-centered therapy: Its current practice, implications and theory. London: Constable. ISBN 1-84119-840-4

Rogers, Carl. (1951). Client-Centered Therapy. p. 64

Sell, H. and Nagpal, R.( 1992) Assessment of subjective wellbeing. New Delhi, World Health Organization,. 
Effect of Spousal Appraisal of Identity Consistency on Well-being of Married Females

Shah, A. ( 1995). Clinical validity of marital quality scale. NIMHANS Journal. 13 ( I), 23- 31.

Suh, E. M. (2002). Culture, identity consistency, and subjective well-being. Journal of Personality and Social Psychology, 83, 1378-1391

Verma, S.K. \& Verma, A. (1989). PGI General Well-being Measure. Ankur Psychological Agency.

Whitbourne, S. K. (1986). The me I know: A study of adult Identity, New york, $N$ Y: Springerverlag.

How to cite this article: Ghosh S, Mukhopadhyay P (2016), Effect of Spousal Appraisal of Identity Consistency on Well-being of Married Females, International Journal of Indian Psychology, Volume 4, Issue 1, No. 81, ISSN:2348-5396 (e), ISSN:2349-3429 (p), DIP:18.01.126/20160401, ISBN:978-1-365-59365-9 